\title{
BIATRIAL THROMBOEMBOLISM WITH PARADOXICAL EMBOLISM THROUGH PERSISTENT FORAMEN OVALE
}

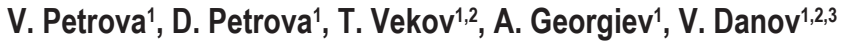 \\ ${ }^{1}$ MHAT Heart and Brain - Pleven, Bulgaria \\ ${ }^{2}$ Medical University - Pleven, Bulgaria \\ 3University Ruse „Angel Kanchev“ - Ruse, Bulgaria
}

\begin{abstract}
We present a case of a biatrial thrombus penetrating through the PFO against the background of massive bilateral pulmonary thromboembolism in a 75-year-old patient. Because of the rare condition, there are no literature data on consensus treatment. Due to the patient's clinical status and the results of the diagnostic tests, for the specific clinical case was made a decision for emergency surgical thrombectomy with PFO closure.
\end{abstract}

Key words: Pulmonary thromboembolism, persistent foramen ovale (PFO), thrombus, paradoxical embolism

Corresponding author: V. Petrova, MHAT Heart and Brain, 2 Pier Curi Str., 5800 Pleven, Bulgaria, e-mail: vanq_yurieva@abv.bg

\section{INTRODUCTION}

$\mathrm{P}$ ulmonary thromboembolism (PTE) is a phase of a pathologic continuum - peripheral vein thrombus formation, embolization of the thrombus and its migration to the pulmonary artery. In rare cases, PTE is associated with an increased risk of ischemic stroke, with the underlying mechanism being paradoxical embolism through persistent foramen ovale (PFO) [1]. The frequency of ischemic stroke in patients with symptomatic PTE is higher in those with PFO than in those without PFO [2]. The PFO is found in approximately $25 \%$ of the total population. Pulmonary embolism (PE) causes an increase in right atrium pressure which in combination with PFO could lead to the appearance of right to left shunt and paradoxical embolism [2].

As such cases are rare there is no consensus on the treatment strategy of pulmonary thromboembolism with trapped thrombus in PFO. Therapeutic options include anticoagulant therapy, surgical treatment (thrombectomy), possibly with PFO closure, an inferior cava filter insertion [3].

\section{CASE REPORT}

A 75-year-old lady was admitted to our department with a recent history of progressive breathlessness on exertion and unspecific chest pain.

The patient had a history of hypertension, permanent atrial fibrillation with no anticoagulation, bronchial asthma, diabetes mellitus type 2 , bilateral gonarthrosis and obesity.

Physical findings included perioral and peripheral cyanosis, jugular vein distention, prolonged expiration and wheeze; tachyarythmic heart rate of 115 beats/ min; arterial blood pressure of $90 / 60 \mathrm{~mm} \mathrm{Hg}$. Liver was palpable $3 \mathrm{~cm}$ below right costal margin. Lower limbs were with pretibial oedema and normal peripheral artery pulsations. 
Serial 12-lead ECG showed atrial fibrillation with a ventricular rate of 115 beats per minute, right bundle branch block, deep S-waves in the precordial leads.

Laboratory blood tests revealed slightly elevated serum troponin I and D-dimers (> $500 \mathrm{ng} / \mathrm{ml}$ ) and normal CKP and CKP-MB levels. Arterial blood gas analysis showed hypoxemia and hypocapnia.

Chest X-ray showed elevated diaphragm bilaterally, proximal pulmonary arteries distention, normal cardiac contour, no pulmonary infiltrates

Both transthoracic (TTE) and transesophageal (TEE) echocardiography were performed and revealed large thrombotic mass in both atria crossing through patent foramen ovale, dilated right ventricle with presence of McConnell's sign, moderate tricuspid valve regurgitation, dilated pulmonary artery trunk with systolic pressure of $45 \mathrm{mmHg}$ (Fig. $1 \mathrm{~A}, \mathrm{~B}$ ).

Computer tomographic (CT) pulmonary angiography was requested and confirmed the presence of proximal bilateral main pulmonary artery emboli, thrombus in transit through a patent foramen ovale, lobar, segmental and subsegmental bilateral pulmonary thromboembolism (Fig. 3 A, B).
Thrombus-in-transit through PFO to left atrium (LA) was considered a contraindication for trombolytic therapy because of the high risk of fragmentation and following paradoxal arterial embolism. In hospital and 30 day mortality scores were calculated: PESI V class - 185; sPESI - 4. Poor clinical condition, hemodynamic instability and the high risk of early death indicated urgent surgical embolectomy despite high perioperative risk (Euro score II 66.6\%).

The patient was transferred to the operating theatre. After median sternotomy, normothermic CPB was initiated, which immediately improved the hemodynamic condition of the patient. The arterial return of the CPB machine was designed to go both to ascending aorta and left atrium with the atrial cannula kept clamped. After cardioplegic arrest, the right atrium was incised and the right atrial aspect of the thrombus attached to the PFO (the thrombus-in-transit was found prominating through the mitral valve into the the left ventricle) Fig. 4 (A). PFO was closed by a direct suture. Left atrial appendage was also sutured to reduce the risk from thromboembolism and stroke. A longitudinal incision was made in the pulmonary artery trunk distal to the pulmonary valve and was extended to the proximal

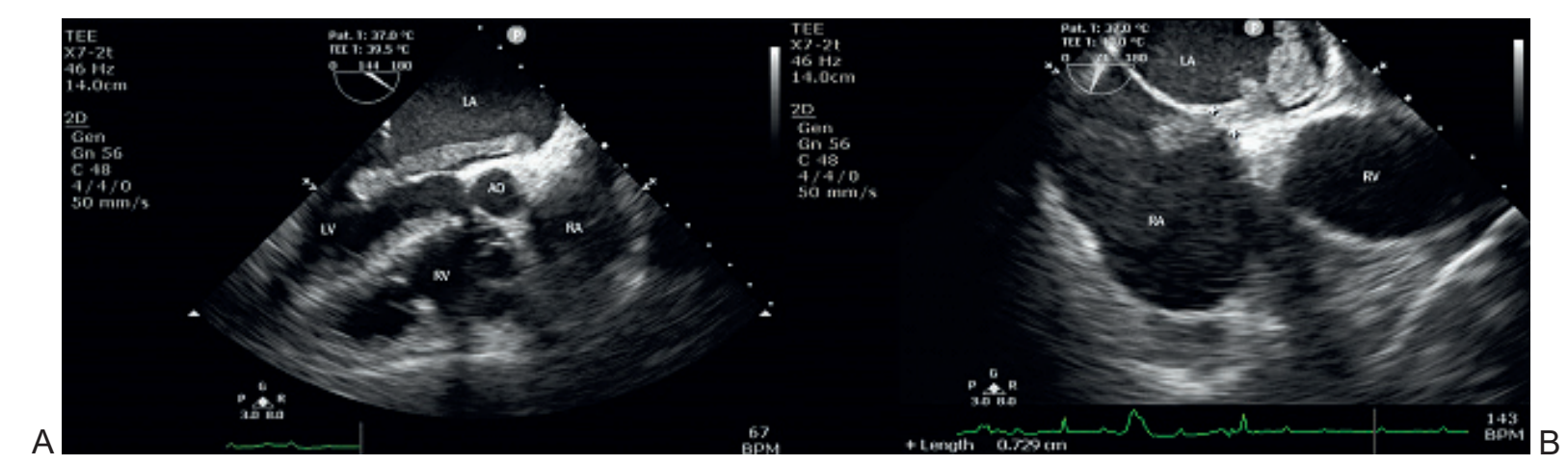

Fig. 1. Preoperative TEE. The thrombus in the left heart was found to be actively mobile and partially prolapsing across the mitral valve to the LV (A). High esophageal views showed a large thrombus entrapped in patent foramen ovale and propagating from right to the left atrium $(B)$
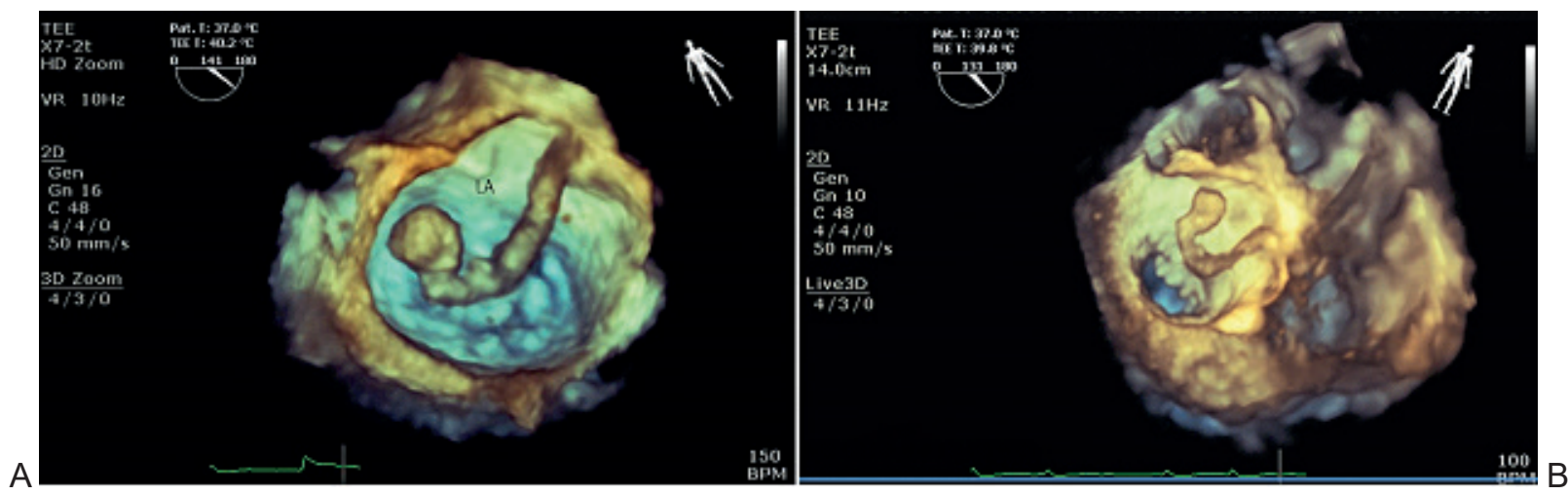

Fig. 2. Preoperative i3D-TTE images showing a large thrombus-in-transit across the PFO (A, B) 


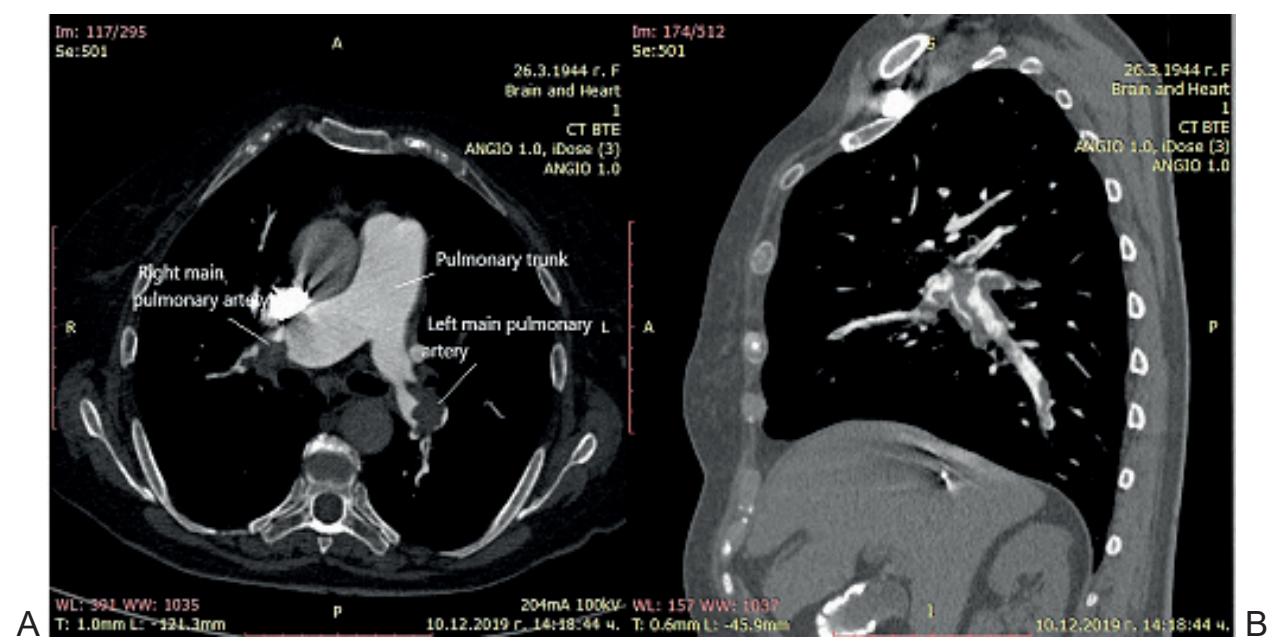

Fig. 3. Chest CT showing a saddle thrombus lodged in the bifurcation of the pulmonary trunk. Massive clot burden and near complete occlusion of the left main and branch pulmonary arteries (A) Multiple filling defects of segmental and subsegmental pulmonary arteries corresponding to the superior, middle and inferior lung lobes $(B)$

right and left pulmonary artery branches. The thrombotic material was extracted by means of forceps and suction (Fig. 4 B). Then with the pulmonary artery was still open, the clamp on the left atrial cannula was released. Blood filled the left atrium and began flowing into the pulmonary artery in a retrograde fashion. The lungs were repeatedly inflated and manually compressed in order to mobilize any residual fragment of thrombotic material that may be lodged in the distal branches of the pulmonary artery and to facilitate the elimination of residual air bubbles. The clots were aspirated, and all air was progressively eliminated from the pulmonary circulation. The pulmonary artery was then closed, and the left atrial cannula was disconnected again. The aortic cross-clamp was released and weaning of CPB was achieved with of epinephrine and dobutamine infusions.

Patient's cardiac and respiratory functions improved immediately after surgery. Early anticoagulant thera- py with i.v. heparin was initiated on 3rd postoperative hour. Weaning of catecholamines and mechanical ventilation was achieved on 4th postoperative day. On the 8th postoperative day oral anticoagulation was commenced. Postoperative lower limb Doppler ultrasonography detected deep vein thromboses of the left femoral and iliac veins.

No perioperative complications were observed. The patient had an uneventful recovery and was discharged home on the 28th postoperative day.

Histological verification showed mixed thrombus.

\section{DISCUSSION}

PFO is one of the most common congenital heart defects, however, prolapsing thrombus through a PFO is a rare complication of pulmonary embolism and is associated with high mortality. The transient increase in right atrial pressure results in a right-to-left shunt

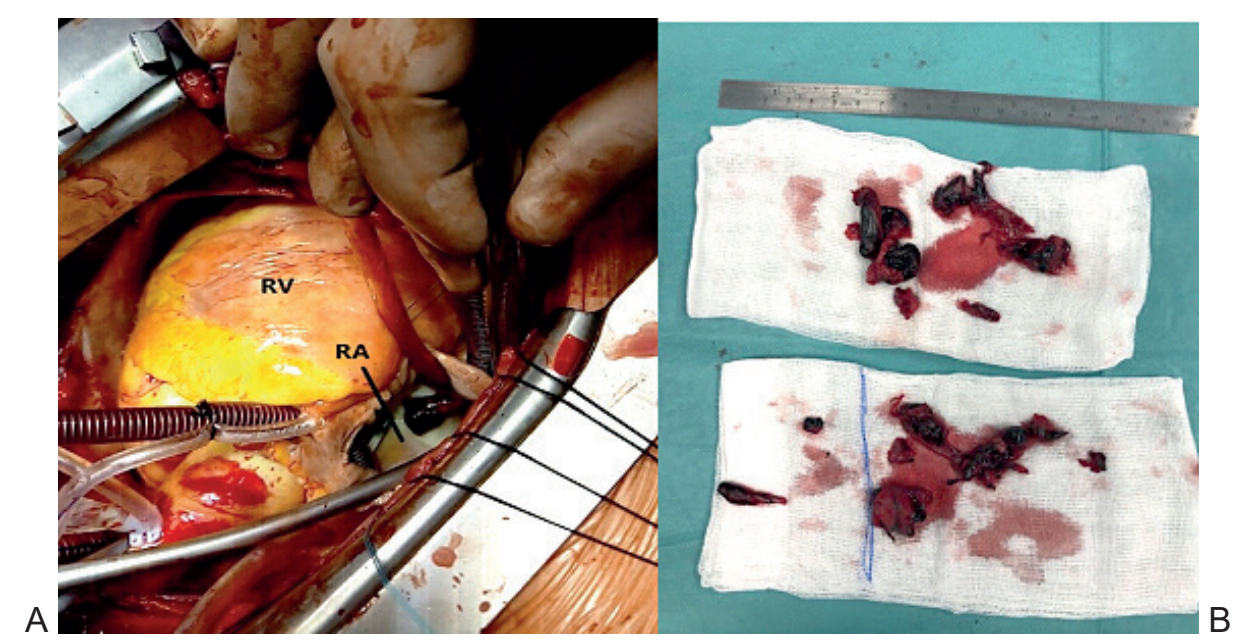

Fig. 4. Part of the thrombus in the right atrium. The patient had right atriotomy and pulmonary arteriotomy (A) with extraction of a large amount of thrombotic material that obstructed the pulmonary arteries (B) 
resulting in paradoxical thromboembolism through PFO [4].

Paradoxical embolism causes $2 \%$ of arterial embolization. Studies on proliferative thrombus in PFO that would provide direct evidence of paradoxical embolism are scarce and there is no consensus on treatment. Therapeutic options are cardiac surgery or thrombolysis [5]. Thrombolysis is a fast, widespread and effective treatment, but it bears the risk of thrombus dislocation with paradoxical arterial embolism and irreversible ischemia [6]. Some systematic retrospective reviews have shown that surgical treatment compared to anticoagulation or thrombolysis is associated with a significantly lower 60-day mortality rate and a lower percentage of embolic events compared to other treatment options [5, 6]. Thrombolysis or anticoagulation are equally acceptable therapeutic alternatives in patients with hemodynamic instability and in the absence of contraindications [7].

\section{CONCLUSION}

Biatrial thrombus penetrating through the PFO and extending through the mitral valve into the left ventricle combined with massive bilateral thromboembolism is a life-threatening condition requiring immediate treatment. The lack of a single consensus makes it difficult to choose between fibrinolysis or surgical embolectomy in the absence of contraindications. We believe that urgent surgical thrombectomy with PFO closure, despite its potential complications, is the superior treatment option to decrease mortality in such cases.

Disclosure summary: The authors have nothing to disclose.

\section{REFERENCES:}

1. Le Moigne E, Timsit S, Ben Salem D, et al. Patent foramen ovale and ischemic stroke in patients with pulmonary embolism [published online May 7, 2019]. Ann Intern Med. doi: 10.7326/M18-3485.

2. Windecker S, Stortecky S, Meier B. Paradoxical embolism. J Am Coll Cardiol. 2014 Jul 29. 64 (4):403-15.

3. Jeremiah haines, Amir Darki, Carlos Bechara, Jason Frazier and $r$. Anthony Perez-Tamayo Management of acute pulmonary embolism in the presence of a patent foramen ovale and subsequent paradoxical embolism 2019, 73, 9 Supplement 1, 2504.

4. Grander W, Schachner T, Velik-Salchner C. et al. Patent foramen ovale and major pulmonary embolism. J Cardiothorac Vasc Anesth. 2011; 25: 841-843.

5. Seo WW, Kim SE, Park MS, Lee JH, Park DG, et al. (2017) Systematic Review of Treatment for Trapped Thrombus in Patent Foramen Ovale. Korean Circ J 47: 776-785.

6. Worku B, Gulkarov I, Steele J. et al. Paradoxical embolus in transit. Ann Thorac Surg. 2014; 98: 717-719.

7. Erkut B, Sevimli S, Ates A. et al. Entrapped thrombus in a patent foramen ovale: Complicated by pulmonary embolism without paradoxical embolism. Tex Heart Inst J. 2008; 35: 371-372.

Approved: 06 May 2020, Last revision: 05 August 2020 